\title{
Onset and Duration of Protective Immunity in Challenged Volunteers after Vaccination with Live Oral Cholera Vaccine CVD 103-HgR
}

\author{
Carol O. Tacket, Genevieve Losonsky, James P. Nataro, \\ Stanley J. Cryz, Robert Edelman, James B. Kaper, and \\ Myron M. Levine
}

\author{
Center for Vaccine Development, Division of Geographic Medicine, \\ Department of Medicine, University of Maryland School of Medicine, \\ Baltimore, and Swiss Serum and Vaccine Institute, Berne, Switzerland
}

CVD 103-HgR is a live oral cholera vaccine that, in phase I and II studies to date, has been well tolerated and immunogenic. In challenge studies of US volunteers conducted 4-5 weeks after vaccination, CVD $103-\mathrm{HgR}$ provided significant protection against experimental cholera due to classical and El Tor Vibrio cholerae O1. To determine the onset and duration of protection, two volunteer challenge studies were conducted: the first, 6 months after vaccination and the second, 8 days after vaccination. In both studies, CVD 103-HgR was $100 \%$ protective against diarrhea and significantly reduced the rate of shedding of vibrios after challenge with $V$. cholerae classical Inaba strain 569B, the virulent parent strain of CVD 103-HgR. Previously vaccinated subjects were less likely than naive controls to develop rises in titer of vibriocidal antibodies after challenge $(P=.002)$, and the mean peak titer of vibriocidal antibodies was less than among controls. CVD 103-HgR can provide homologous protective immunity as soon as 8 days after vaccination and protection can persist for at least 6 months.

The expansion of the seventh pandemic of cholera into South America has increased the urgency to develop a safe, effective, and long-lasting cholera vaccine. A vaccine that is rapidly effective after a single dose and that could be given orally would be especially desirable because of the ease of administration to residents of remote areas and its usefulness soon after cholera is introduced into a community.

CVD $103-\mathrm{HgR}$ is a live oral Vibrio cholerae $\mathrm{Ol}$ vaccine strain that meets these criteria. The strain was developed by deleting $94 \%$ of the $c t x A$ gene and introducing a gene encoding resistance to mercury into a chromosomal locus (hlyA) of V. cholerae classical Inaba strain 569B [1]. In phase I and II studies in North American, European, Thai, and Peruvian adults and Indonesian and Peruvian children, this vaccine has been well tolerated and in those without preexisting vibriocidal antibodies, highly immunogenic [2-4] (unpublished data). In volunteer challenge studies of North Americans conducted $4-5$ weeks after vaccination, CVD $103-\mathrm{HgR}$ protected against experimental challenge with both $\mathrm{El}$ Tor and classical biotypes of $V$. cholerae $\mathrm{Ol}$ [2]. The interval of 4-5 weeks between vaccination and challenge was chosen on the basis of the likely timing of mature immune responses and

Received 13 April 1992; revised 20 May 1992.

Written informed consent was obtained from the volunteers enrolled in these studies, and the buman experimentation guidelines of the US Department of Health and Human Services and those of the University of Maryland at Baltimore were followed in the conduct of the clinical research.

Financial support: National Institutes of Health (Al-15096) and Swiss Serum and Vaccine Institute.

Reprints or correspondence: Dr. Carol O. Tacket, Center for Vaccine Development, 10 S. Pine St., Baltimore, MD 21201.

The Journal of Infectious Diseases 1992;166:837-41 (C) 1992 by The University of Chicago. All rights reserved. $0022-1899 / 92 / 6604-0021 \$ 01.00$ practical expedience in maintaining a cohort of vaccinees willing to undergo challenge. Moreover, this was the interval used in previous studies that demonstrated the effectiveness of infection-derived and vaccine-derived immunity to cholera $[2,5-8]$.

We wished to further explore the limits of protective immunity elicited by CVD $103-\mathrm{HgR}$ in terms of how soon after vaccination protective responses appear and how long these responses persist. These issues are especially important because a vaccine with rapid onset of protection (i.e., within days of vaccination) would be an extremely valuable tool for the control of epidemic cholera as soon as an outbreak is detected. In addition, a vaccine that elicits protective responses of long duration would be desirable for control of endemic cholera. Finally, a medium-range duration of protection (e.g., months) would be desirable in a vaccine for use by travelers. We report the results of volunteer challenge studies conducted in two cohorts of adult volunteers vaccinated with CVD 103-HgR 8 days or 6 months before homologous $V$. cholerae challenge to approximate the expected level of protective efficacy in the field soon after vaccination and within the subsequent 6 months.

\section{Materials and Methods}

Volunteers. Healthy adults 18-39 years old from the Baltimore community were recruited to participate in a 6-month or an 8-day postvaccination challenge study. Volunteers were screened to confirm their good health by medical histories and physical examinations, a battery of clinical hematologic and chemistry tests, electrocardiograms, and serologic tests for syphilis, hepatitis B surface antigen, and human immunodeficiency virus. Before vaccination and challenge, stools were examined for bacterial pathogens, ova, and parasites. 
Vaccine. V. cholerae vaccine strain CVD $103-\mathrm{HgR}$ was grown, lyophilized, and packaged at the Swiss Serum and Vaccine Institute. The formulation consisted of sachets containing a single dose (3-5 $\times 10^{8}$ or 3-5 $\times 10^{9}$ viable organisms) of lyophilized vaccine and $25 \mathrm{mg}$ of aspartame. An accompanying sachet contained an effervescent buffer powder consisting of $2.5 \mathrm{~g}$ of sodium bicarbonate and $1.65 \mathrm{~g}$ of ascorbic acid. The vaccine was stored at $4^{\circ} \mathrm{C}$ before use.

Vaccination. Two cohorts of volunteers were recruited for the two challenge studies depending on the time of challenge after vaccination. In the first study, 22 outpatient volunteers received a single oral dose of $\sim 3-5 \times 10^{8} \mathrm{cfu}$ of CVD 103-HgR; 2 months later 7 other volunteers received a single oral dose of $\sim 3-5 \times 10^{9} \mathrm{cfu}$. These volunteers were to be challenged 6 and 4 months after vaccination, respectively, in the same challenge study. The higher dose of vaccine was chosen because preliminary data suggested increased immunogenicity after this dose among vaccinees residing in an endemic area (unpublished data). In the second study, 11 volunteers received $3-5 \times 10^{8} \mathrm{cfu}$ 8 days before challenge.

At the time of vaccination, a sachet of buffer was added to $100 \mathrm{~mL}$ of distilled water in a cup. A sachet containing a dose of vaccine was then added to the buffer to make a vaccine cocktail, which the volunteers ingested. Volunteers had nothing to eat or drink for $90 \mathrm{~min}$ before and after vaccination.

Diarrhea after vaccination was defined as passage of at least four unformed stools over $24 \mathrm{~h}$. Volunteers kept a stool record for 5 days after vaccination and called the Center for Vaccine Development daily for 5 days to report symptoms.

Challenge. In the first study, volunteers vaccinated with $10^{8}$ cfu of CVD 103-HgR 6 months previously $(n=11)$ and volunteers vaccinated with $10^{9} \mathrm{cfu} 4$ months previously $(n=3)$ were admitted to the Research Isolation Ward of the Center for Vaccine Development (University of Maryland Hospital) with a group of unvaccinated control volunteers $(n=15)$. Challenge studies werc done under quarantine administered by the Bureau of Disease Control, Baltimore City Health Department. After 48 h of acclimatization, volunteers received a dose of $4 \times 10^{6} \mathrm{cfu}$ of $V$. cholerae strain 569B. In the second study, volunteers who had received $10^{8} \mathrm{cfu}$ of CVD $103-\mathrm{HgR} 8$ days before challenge were admitted to the Research Isolation Ward, were observed for 48 $h$, and reccived $3 \times 10^{7} \mathrm{cfu}$ of $V$. cholerae strain $569 \mathrm{~B}$.

Volunteers in both studies were closely monitored for 10 days after challenge; vital signs were measured every $6 \mathrm{~h}$. All stools from each volunteer were collected in plastic containers, examined, and graded on a five-point scale [1], and the volume was measured when the stool was loose. Volunteers were interviewed daily by a physician and asked about symptoms. Diarrhea after challenge was defined as two or more loose stools within $48 \mathrm{~h}$ totaling $\geqslant 200 \mathrm{~mL}$ in volume or a single loose stool $\geqslant 300 \mathrm{~mL}$ in volume. Antibiotic therapy (tetracycline, $500 \mathrm{mg}$ every $6 \mathrm{~h}$ for 5 days) was given before discharge.

Bacteriology. Every stool passed by volunteers after challenge was cultured daily for the challenge strain. Stool was plated directly onto thiosulfate-citrate-bile-sucrose (TCBS) agar and into alkaline peptone water enrichment broth for overnight incubation before plating onto TCBS agar. The number of challenge organisms per gram of stool was determined each day for up to two stools.

At $\sim 20$ and $44 \mathrm{~h}$ after challenge, fasting volunteers swal- lowed gelatin capsules containing string devices (Entero-Test; HDC, Mountain View, CA) to collect samples of bile-stained duodenal fluid. After $4 \mathrm{~h}$, the strings were removed and the color and $\mathrm{pH}$ of the distal $15 \mathrm{~cm}$ were recorded. Duodenal fluid was squeezed from the end of the string and cultured as described.

Immunology. For volunteers who would receive a challenge 4-6 months after vaccination, serum samples were obtained before vaccination and on days 10 and 28 after vaccination and just before challenge. For those who would receive the challenge 8 days after vaccination, serum was obtained before vaccination and just before challenge. After challenge, serum was obtained at days 7,21, and 28. Titers of vibriocidal antibodies were measured in serum [9] and antibody responses to $V$. cholerae lipopolysaccharide and cholera toxin were measured by ELISA [10].

\section{Results}

\section{Study 1: Vaccination and Challenge 4-6 Months Later}

Clinical responses to vaccination. No diarrhea or other significant symptoms were reported after vaccination.

Serologic responses to vaccination. After vaccination with a single oral dose of $10^{8} \mathrm{cfu}$ of CVD $103-\mathrm{HgR}, 20(95 \%)$ of 21 vaccinees developed fourfold or greater rises in serum Inaba vibriocidal antibody with a geometric mean peak titer after vaccination of 3561. After vaccination with a single dose of $10^{9} \mathrm{cfu}$, all 7 vaccinees developed rises in vibriocidal antibodies with a peak geometric mean titer of 2826 after vaccination. Among all 28 vaccinees, $19(68 \%)$ had peak vibriocidal reciprocal titers $\geqslant 2560$. After vaccination, 13 (62\%) of 21 vaccinees who received the lower dose and all 7 who received the higher dose developed significant rises in serum IgG antibody against cholera toxin.

Clinical and bacteriologic responses to challenge. Of the 28 volunteers who had been vaccinated $4-6$ months previously, 14 ( 11 who had received $10^{8}$ cfu and 3 who had received $10^{9} \mathrm{cfu}$ ) were available for challenge. After challenge with $V$. cholerae classical Inaba strain 569B, $10(67 \%)$ of 15 control volunteers and none of 14 previously vaccinated volunteers developed diarrhea $(P<.001$, Fisher's exact test $)$. The mean stool volumes, number of loose stools, and mean incubation period are shown in table 1 . All 15 control volunteers and $3(21 \%)$ of 14 vaccinees shed the challenge strain in the stool $(P<.001$, Fisher's exact test $)$; the mean number of organisms shed by vaccinees with positive coprocultures was 10-fold less than that shed by control volunteers (table 1).

Serologic responses to challenge. Before challenge, vaccinees had a significantly higher Inaba vibriocidal antibody titer than did controls (table 2); after challenge, the vibriocidal titer rose in only half the vaccinees while all controls had significant rises in vibriocidal antibody titers $(P=.002$, Fisher's exact test). The peak geometric mean reciprocal titer in vaccinees after challenge (1640) was significantly less than in controls (9778). 
Table 1. Clinical and bacteriologic responses to classical Inaba Vibrio cholerae challenge in recipients of a single dose of CVD 103-HgR given 6 months or 8 days before challenge.

\begin{tabular}{|c|c|c|c|c|c|c|}
\hline $\begin{array}{l}\text { Time of } \\
\text { immunization } \\
\text { before } \\
\text { challenge. } \\
\text { group. } P\end{array}$ & $\begin{array}{c}\text { No. with } \\
\text { diarrhea/ } \\
\text { total }\end{array}$ & $\begin{array}{l}\text { No. (mean) of } \\
\text { loose stools* }\end{array}$ & $\begin{array}{c}\text { Volume }(\mathrm{mL}) \\
\text { of stools } \\
\text { (mean, range) }\end{array}$ & $\begin{array}{l}\text { Incubation } \\
\text { period } \\
(\text { mean } h)\end{array}$ & $\begin{array}{c}\text { No. } \\
\text { shedding } \\
\text { in } \\
\text { stool/total }\end{array}$ & $\begin{array}{c}\text { Peak } \\
\text { excretion } \\
\text { (mean cfu/g) }\end{array}$ \\
\hline \multicolumn{7}{|l|}{ 4-6 months } \\
\hline Vaccinees & $0 / 14$ & 0 & 0 & - & $3 / 14$ & $1.6 \times 10^{3 \dagger}$ \\
\hline Controls & $10 / 15$ & 6.3 & $753(227-2741)$ & 55.9 & $15 / 15$ & $1.5 \times 10^{4}$ \\
\hline$P$ & $<.001^{\ddagger}$ & & & & $<.001^{\ddagger}$ & NS $^{\mathbf{8}}$ \\
\hline \multicolumn{7}{|l|}{8 days } \\
\hline Vaccinees & $0 / 11$ & 0 & 0 & - & $1 / 11$ & $2.0 \times 10^{3}$ \\
\hline Controls & $8 / 11$ & 7.8 & $1059(214-2810)$ & 36.6 & $9 / 11$ & $3.5 \times 10^{4}$ \\
\hline$P$ & $<.001^{\ddagger}$ & & & & $<.002^{\ddagger}$ & NS \\
\hline
\end{tabular}

NOTE. NS, not significant; cfu, colony-forming units. Vaccine efficacy was $100 \%$.

* During 8-day observation.

† Three vaccinees with positive coprocultures.

† Fisher's exact test.

8 test.

\section{Study 2: Vaccination and Challenge 8 Days Later}

Clinical response to vaccination. No diarrhea or significant symptoms were reported by volunteers after vaccination. No vaccine organisms were detected in the stools of volunteers immediately before challenge.

Clinical and bacteriologic response to challenge. After challenge with $V$. cholerae classical Inaba strain 569B, 8 (73\%) of 11 control volunteers and none of 11 previously vaccinated volunteers developed diarrhea $(P<.001$, Fisher's exact test). The mean stool volumes, number of loose stools, and mean incubation period are shown in table 1 . Nine $(82 \%)$ of 11 control volunteers and only $1(9 \%)$ of 11 vaccinees shed the challenge strain in the stool $(P=.002$, Fisher's exact test); the number of organisms shed by the vaccinee was 10 -fold less than that shed by control volunteers (table 1).

Serologic response to challenge. The serologic response to challenge is shown in table 3 . The vaccinees had a mean reciprocal vibriocidal titer of 682 and the controls had a mean titer of 14 on the day of challenge. The vibriocidal titer peaked earlier among vaccinees (day 7 after challenge) than among controls (day 22) and fell more rapidly after challenge (table 3 ).

\section{Discussion}

The ideal cholera vaccine should have the following characteristics: safety, oral delivery for ease of administration,

Table 2. Immune responses in volunteers vaccinated with a single oral dose of CVD $103-\mathrm{HgR}$ and challenged 4-6 months later with Vibrio cholerae classical Inaba strain 569B.

\begin{tabular}{|c|c|c|c|c|c|c|}
\hline & \multicolumn{3}{|c|}{ Vaccinees } & \multicolumn{3}{|c|}{ Controls } \\
\hline & $\begin{array}{l}\text { No. with } \\
\text { responses } \\
\text { after } \\
\text { challenge* }\end{array}$ & $\begin{array}{l}\text { Response } \\
\text { before } \\
\text { challenge }\end{array}$ & $\begin{array}{c}\text { Peak } \\
\text { response } \\
\text { after } \\
\text { challenge }\end{array}$ & $\begin{array}{l}\text { No. with } \\
\text { responses } \\
\text { after } \\
\text { challenge* }\end{array}$ & $\begin{array}{l}\text { Response } \\
\text { before } \\
\text { challenge }\end{array}$ & $\begin{array}{c}\text { Peak } \\
\text { response } \\
\text { after } \\
\text { challenge }\end{array}$ \\
\hline Inaba vibriocidal antibody $(G M T)^{\ddagger}$ & $7 / 14^{\dagger}$ & $276^{\ddagger}$ & $1640^{8}$ & $15 / 15^{t}$ & $66^{\ddagger}$ & $9778^{\S}$ \\
\hline Inaba LPS IgG antibody (GMT) & $7 / 14$ & 69 & 305 & $5 / 15$ & 76 & 168 \\
\hline Cholera toxin IgG antibody (OD) & $6 / 14$ & 0.37 & 0.99 & $15 / 15$ & 0.11 & 1.36 \\
\hline
\end{tabular}

NOTE. GMT, geometric mean titer; LPS, lipopolysaccharide; OD, optical density. Of 14 challenged vaccinees, 11 had received $5 \times 10^{8}$ cfu and 3 had received $5 \times 10^{9} \mathrm{cfu}$ of CVD $103-\mathrm{HgR}$.

* Fourfold rise in antibody titer or significant change in $O D$ after vaccination.

+ Vaccinees vs. controls: $P=.002$. Fisher's exact test.

$¥$ Vaccinees vs. controls: $P=.007$, Mann-Whitney U test.

Vaccinees vs. controls: $P=.004$, Mann-Whitney $U$ test. 
Table 3. Immune responses in volunteers vaccinated with a single oral dose of CVD 103-HgR and challenged 8 days later with Vibrio cholerae classical Inaba strain 569B.

\begin{tabular}{|c|c|c|c|c|c|}
\hline & \multirow{2}{*}{$\begin{array}{l}\text { Vaccination, } \\
-8 \text { days }\end{array}$} & \multicolumn{4}{|c|}{ Challenge day } \\
\hline & & 0 & 7 & 22 & 28 \\
\hline \multicolumn{6}{|l|}{ Vaccinees } \\
\hline Vibriocidal antibody (GMT) & 15 & 682 & 2119 & 597 & 422 \\
\hline Inaba LPS IgG (GMT) & 22 & 23 & 35 & 35 & 29 \\
\hline Cholera toxin IgG (OD) & 0.18 & 0.46 & 0.73 & 0.59 & 0.61 \\
\hline \multicolumn{6}{|l|}{ Controls } \\
\hline Vibriocidal antibody (GMT) & NA & 14 & 1280 & 1940 & 854 \\
\hline Inaba LPS IgG (GMT) & NA & 29 & 55 & 92 & 80 \\
\hline Cholera toxin IgG (OD) & NA & 0.07 & 0.37 & 1.10 & 1.03 \\
\hline
\end{tabular}

NOTE. GMT, geometric mean titer; OD, optical density; LPS, lipopolysaccharide; NA, not applicable.

requirement for only a single dose, high protective efficacy, a long duration of protection, and a rapid onset of protection after vaccination. In volunteer challenge studies to date, CVD 103-HgR has met these criteria. The maximum duration of protection elicited by CVD 103-HgR is unknown, but the hope is that CVD 103-HgR will perform like other live attenuated vaccines in providing many years of protective efficacy.

In these volunteer studies, the challenge strain chosen (classical Inaba strain 569B) was the virulent parent strain of CVD 103-HgR. Clearly, the choice of the biotype-homologous strain for challenge increased the likelihood of showing protection by CVD 103-HgR. In two previous challenge studies in which CVD 103- $\mathrm{HgR}$ vaccinees received challenges with biotype-heterologous El Tor Inaba or El Tor Ogawa, $\sim 65 \%$ protective efficacy was seen $4-5$ weeks after vaccination [2] (unpublished data). The onset and duration of biotype heterologous protection will be determined in future field efficacy trials.

These studies also confirmed an important serologic observation originally made in phase II studies among residents of developing areas. In Thai soldiers, overall seroconversion rates to CVD $103-\mathrm{HgR}$ were lower than in US and European volunteers, in large part because Thais had elevated, sometimes high, vibriocidal titers before vaccination [4]. In our cohort of US volunteers vaccinated 6 months before challenge, many of whom retained elevated vibriocidal titers at the time of challenge, the rate of seroconversion after challenge was half that seen in naive control volunteers $(P=$ $.002)$. Vibriocidal antibody may be an epidemiologic correlate of immunity [11-13], but rates of seroconversion after vaccination among populations with high levels of preexisting vibriocidal antibody may not correlate with or predict vaccine efficacy [4]. In other words, failure to manifest seroconversion of vibriocidal antibody after vaccination with CVD $103-\mathrm{HgR}$ in persons who already have elevated titers before vaccination does not signify lack of immunogenicity. To the contrary, it appears that individuals with immunity evidenced by elevated serum vibriocidal titers often are not further boosted after vaccination with CVD $103-\mathrm{HgR}$ or exposure to wild-type $V$. cholerae $\mathrm{OI}$.

We conclude that the cholera vaccine CVD $103-\mathrm{HgR}$ is likely to be successful for control of epidemic cholera and cholera among travelers because of its rapid onset of protection. Its usefulness in endemic areas will be measured in upcoming field studies.

\section{Acknowledgments}

We acknowledge the assistance of Kathleen Palmer, Brenda Berger, and Will Willis in recruiting and retaining volunteers for this study, Sylvia O'Donnell for research coordination, and Steven S. Wasserman for statistical assistance. Yu Lim, Mardi Reymann, and Linda Guers provided technical laboratory support. We also recognize the invaluable contribution of the volunteers who participated in these studies.

\section{References}

1. Kaper JB, Lockman H, Baldini MM, Levine MM. A recombinant live oral cholera vaccine. Bio/Tech 1984;2:345-9.

2. Levine MM, Kaper JB, Herrington D, et al. Safety, immunogenicity, and efficacy of recombinant live oral cholera vaccines, CVD 103 and CVD 103-HgR. Lancet 1988;2:2467-70.

3. Cryz SJ Jr, Levine MM, Kaper JB, Furer E, Althaus B. Randomized double-blind placebo controlled trial to evaluate the safety and immunogenicity of the live oral cholera vaccine strain CVD $103-\mathrm{HgR}$ in Swiss adults. Vaccine 1990;8:577-80.

4. Su-Arehawaratana P, Singharaj P, Taylor DN, et al. Safety and immunogenicity of different immunization regimens of CVD 103-HgR live oral cholera vaccine in soldiers and civilians in Thailand. $J$ Infect Dis 1992;165:1042-8.

5. Levine MM, Nalin DR, Craig JP, et al. Immunity of cholera in man: relative role of antibacterial versus antitoxic immunity. Trans $\mathrm{R}$ Soc Trop Med Hyg 1979;73:3-9.

6. Levine MM, Kaper JB, Black RE, Clements ML. New knowledge on 
pathogenesis of bacterial enteric infections as applied to vaccine development. Microbiol Rev 1983;47:510-50.

7. Levine MM, Black RE, Clements ML, et al. Evaluation in man of attenuated Vibrio cholerae El Tor Ogawa strain Texas Star-SR as a live oral vaccine. Infect Immun 1984;43:515-22.

8. Levine MM, Kaper JB, Herrington DA, et al. Volunteer studies of deletion mutants of Vibrio cholerae $\mathrm{Ol}$ prepared by recombinant techniques.

9. Clements ML, Levine MM, Young CR, et al. Magnitude, kinetics and duration of vibriocidal antibody response in North Americans after ingestion of Vibrio cholerae. J Infect Dis 1982;145:465-73.

10. Levine MM, Young CR. Black RE. Takeda Y, Finkelstein RA. Enzyme-linked immunosorbent assay to measure antibodies to purified heat-labile enterotoxins from human and porcine strains and to cholera toxin: application in serodiagnosis and seroepidemiology. J Clin Microbiol 1985;21:174-9.

11. Mosley WH. The role of immunity in cholera. A review of epidemiological and serological studies. Texas Rep Biol Med 1969;27:227-44.

12. Mosley WH, McCormack WM, Ahmed A, et al. Report of the 1966-67 cholera vaccine field trial in rural East Pakistan. 2. Results of the serologic surveys in the study population-the relationship of case rate to antibody titre and an estimate of the inapparent infection rate with Vibrio cholerae. Bull WHO 1969;40:187-97.

13. Clemens JD, van Loon F. Sack DA, et al. Field trial of oral cholera vaccines in Bangladesh: serum vibriocidal and antitoxic antibodies as markers of the risk of cholera. J Infect Dis 1991:163:1235-42. 